\title{
Associação entre 0 Uso de Sulfoniluréias por Gestantes Diabéticas e Malformaçōes Fetais
}

The Use of Sulfonylureas by Pregnant Diabetic Women and Fetal Malformations

Renan Magalhães Montenegro Junior, Glória Maria Guimarães Ferreira Paccola, Claúdia Martins Faria, Sérgio Pereira da Cunha, Milton Cesar Foss

\begin{abstract}
RESUMO
Objetivo: avaliar a relação entre o uso de sulfoniluréias (SF) por gestantes diabéticas seguidas na nossa clinica e as malformações (MF) fetais detectadas.

Métodos: estudamos, retrospectivamente, 35 gestantes diabéticas tipo 2, seguidas no Ambulatório de Pré-Natal Patológico do Hospital das Clínicas da Faculdade de Medicina de Ribeirão Preto, USP, no periodo de 1993-1995, das quais 22 encontravam-se em uso inadvertido de SF no $1^{\circ}$ trimestre da gestação (grupo SF). Avaliamos a prevalência de $M F e$ comparamos com as gestantes diabéticas que nesse periodo encontravam-se em uso de insulina ou apenas dieta (grupo C). Foram também analisadas outras variáveis: tempo de doença, idade, controle metabólico e seguimento pré-natal.

Resultados: não houve diferença estatisticamente significativa entre os 2 grupos quanto a faixa etária, tempo de evolução do diabetes, controle metabólico ou precocidade do início do pré-natal, sendo a freqüência de $M F$ semelhante nos 2 grupos (8,3\% no grupo C e 13,6\% no grupo SF). As malformações encontradas foram: no grupo SF - agenesia renal, hipoplasia pulmonar e gônadas em fita (paciente 1); membros curtos e pododáctilos de implantação anormal (paciente 2); fenda palatina, implantação baixa de orelhas, pescoço alado, prega palmar única e nariz em sela (paciente 3), e no grupo $C$ - micrognatia, orelha displásica, polidactilia, imperfuração anal, hipospadia, comunicação inter-atrial e comunicação interventricular (paciente 4).

Conclusões: esses dados não nos permitem atribuir as malformações encontradas no grupo SF ao uso de sulfoniluréias, embora nesse grupo tenham ocorrido alterações não usualmente descritas na embriopatia diabética.
\end{abstract}

PALAVRAS-CHAVE: Diabetes mellitus. Malformações fetais.

\section{Introdução}

Apesar das sulfoniluréias (SF) serem amplamente utilizadas em alguns países como Índia, México e África do Sul, têm sido rotineiramente contra-indicadas, no nosso meio, pelos

Divisão de Endocrinologia e Metabologia do Departamento de Clínica Médica e Departamento de Ginecologia e Obstetrícia, Faculdade de Medicina de Ribeirão Preto, USP.

Correspondência:

Milton Cesar Foss

Divisão de Endocrinologia e Metabologia, Departamento de Clínica Médica, Faculdade de Medicina de Ribeirão Preto, USP

Av. dos Bandeirantes 3.900

14049-900 - Ribeirão Preto - SP

Fone: (16) 633.2563 Fax: (16) 633.1144 possiveis efeitos deletérios sobre o desenvolvimento fetal. Sabendo-se que esses antidiabéticos orais atravessam a placenta, tem-se interrogado sobre um possivel efeito teratogênico. Os resultados de estudos anteriores são conflitantes quanto a esse fato. Apesar de ter sido encontrada uma maior prevalência de malformações fetais (MF) em diabéticas em uso de sulfoniluréias, em nenhum desses estudos se pôde estabelecer uma relação causa-efeito ${ }^{1,2,3,4,5,6,7,8}$, devido principalmente à elevada freqüência de mau controle metabólico materno nesses estudos. Há indicações mais recentes de que esta associação não se relaciona ao uso dessas drogas e sim ao mau controle glicêmico ${ }^{8}$. 
Embora as diabéticas tipo 2 usualmente estejam fora da idade reprodutiva, a ocorrência de gestações nessas pacientes em nosso meio tem se mostrado freqüente. Como as sulfoniluréias constituem a terapêtica medicamentosa mais utilizada nesse grupo, sendo elevada a incidência de gestações não-programadas em diabéticas, tem sido comum encontrarmos pacientes em uso dessas drogas no início do seguimento pré-natal.

Desse modo, o objetivo do presente estudo foi avaliar a possivel relação do uso de SF por gestantes diabéticas seguidas na nossa clínica e as malformações fetais detectadas.

\section{Pacientes e Métodos}

Avaliamos, retrospectivamente, 35 casos de gestantes diabéticas tipo 2 seguidas no Ambulatório de Pré-Natal Patológico do Hospital das Clínicas da Faculdade de Medicina de Ribeirão Preto, USP, no período de 1993 a 1995. Anteriormente à gestação, 12 dessas gestantes eram tratadas com dieta ou insulina (grupo C) e 23 usavam sulfoniluréias, sendo que dessas, 22 estiveram em uso da medicação durante $\mathrm{o} 1^{\circ}$ trimestre da gestação (grupo SF).

A duração da utilização das sulfoniluréias pelas gestantes do grupo SF variou de 6 a 28 semanas. A droga foi suspensa na primeira consulta pré-natal, durante a qual todas as pacientes recebiam orientação dietética, sendo associada insulina se necessário.

As pacientes faziam seguimento periódico, com intervalos de 1 a 2 semanas entre os retornos, sendo internadas se apresentassem um controle metabólico ruim. O controle glicêmico foi avaliado pela mediana das glicemias de jejum e pósprandiais coletadas em todas as consultas e internações. Eram colhidas glicemias às 7, 10, 14, 20 e 2 horas. Os parâmetros de controle adequado utilizados foram os seguintes: glicemia de jejum $<105 \mathrm{mg} / \mathrm{dl}$; pré-prandial e pós-prandial $<120 \mathrm{mg} /$ $\mathrm{dl}$; noturna $(2 \mathrm{~h})$ entre 60 e $120 \mathrm{mg} / \mathrm{dl}^{9,10}$.

O acompanhamento obstétrico e endocrinológico e os critérios terapêuticos do diabetes aplicados aos dois grupos eram semelhantes. O pediatra da Unidade de Neonatologia acompanhou todos os partos. Os recém-nascidos (RN) foram examinados imediatamente após o nascimento e avaliados quanto ao estado metabólico e à existência de malformações, com atenção especial para as anormalidades usualmente encontradas em filhos de diabéticas. Em casos suspeitos eram ainda avaliados pelo geneticista.

Os dois grupos foram analisados quanto a idade, tempo de diagnóstico de diabetes, idade gestacional no início do pré-natal, controle glicêmico e incidência de malformações.

No grupo SF analisamos ainda a correlação das MF com as sulfoniluréias utilizadas. As malformações foram divididas em maiores e menores. Consideramos malformações maiores as que resultam em óbito ou morbidade prolongada ou que necessitem de correção cirúrgica. As malformações menores compreendem as restantes ${ }^{8}$.

$\mathrm{Na}$ análise da freqüência de malformações (MF) nos grupos foi utilizado o teste exato de Fisher. Para comparação das médias de idade, tempo de diagnóstico de diabetes, idade gestacional no início do pré-natal, medianas de glicemia de jejum e pósprandial foi utilizado o teste de Wilcoxon-MannWhitney, sendo expressos como médias e desvios padrão (DP). O nivel de significância adotado foi de $5 \%$.

\section{Resultados}

Dentre as 35 diabéticas tipo 2 avaliadas, 4 $(11,4 \%)$ tiveram fetos com malformações, conforme descrito na Tabela 1. Dessas, havia $3 \mathrm{RN}$ de diabéticas que fizeram uso de SF na gestação (grupo $\mathrm{SF}$ ) e um de paciente tratada com dieta e insulina (grupo C).

Tabela 1 - Freqüência de malformações nos grupos sulfoniluréia e controle.

\begin{tabular}{lccc}
\hline & Grupo SF & Grupo C & Total de Pacientes \\
\hline MF maiores & 13,6 & 8,3 & 11,4 \\
MF menores & 9,1 & 8,3 & 8,6 \\
\hline
\end{tabular}

Não houve diferença significativa entre as gestantes diabéticas que estiveram ou não em uso de $\mathrm{SF}$ na gestação quanto a idade $(33,5 \pm 5,3$ vs. $34,4 \pm 5,6$ anos), duração do diabetes $(45,8 \pm 28,4$ vs. 44,9 $\pm 41,0$ meses), idade gestacional ao início do pré-natal ( $15,3 \pm 6,7$ vs. $20,9 \pm 8,2$ semanas) ou controle glicêmico - glicemia de jejum $(125,0 \pm 31,6$ vs. $109,5 \pm 20,3 \mathrm{mg} / \mathrm{dl})$ e pós-prandial $(155,0 \pm$ $33,5$ vs. $142,4 \pm 34,5 \mathrm{mg} / \mathrm{dl})$, conforme mostrado na Tabela 2 . 
Tabela 2 - Características clínicas dos grupos estudados (média \pm desvio padrão).

\begin{tabular}{lrr} 
& & Grupo C \\
\hline Idade (anos) & Grupo SF & $34,4 \pm 5,6$ \\
Duração do diabetes (meses) & $33,5 \pm 5,3$ & $44,9 \pm 41,0$ \\
Idade gestacional ao início do pré-natal (semanas) & $45,8 \pm 28,4$ & $20,9 \pm 8,2$ \\
Suspensão da sulfoniluréia (semanas) & $15,3 \pm 6,7$ & - \\
Medianas das glicemias de jejum (mg/dl) & $11,6 \pm 5,8$ & $109,5 \pm 20,3$ \\
Medianas das glicemias pós-prandiais (mg/dl) & $125,0 \pm 31,6$ & $142,4 \pm 34,5$ \\
\hline
\end{tabular}

SF = sulfoniluréia; $\mathrm{C}=$ controle

Do grupo SF, 15 estiveram em uso de clorpropamida e 7 tomavam glibenclamida, com uma dose que variava de 250 a $500 \mathrm{mg} /$ dia e 5 a $15 \mathrm{mg} /$ dia, respectivamente. As malformações encontradas nos dois fetos das pacientes que usavam clorpropamida foram: agenesia renal, gônadas em fita, hipoplasia pulmonar, membros curtos (MF maiores) e implantação anormal do $3^{\circ}$ pododáctilo (MF menor). O recém-nascido da paciente em uso de glibenclamida apresentava fenda palatina (MF maior), nariz em sela, pescoço alado, prega palmar única e implantação baixa de orelhas (MF menores). O RN da gestante do grupo C apresentava comunicação interventricular (CIV), comunicação inter-atrial (CIA), imperfuração anal e hipospadia (MF maiores), micrognatia, um dedo supranumerário e orelhas displásicas (MF menores) (Tabela 3).

Tabela 3 - Características das pacientes com recém-nascidos malformados e descrição das malformações

\begin{tabular}{|c|c|c|c|c|}
\hline & Paciente 1 & Paciente 2 & Paciente 3 & Paciente 4 \\
\hline Medicação utilizada & clorpropamida & clorpropamida & glibenclamida & insulina \\
\hline Duração do diabetes & 3 anos & 3 anos & 6 anos & 6 anos \\
\hline Suspensão da sulfoniluréia (IG) & 15 semanas & 16 semanas & 9 semanas & - \\
\hline Mediana da glicemia de jejum (mg/dl) & $123 \mathrm{mg} / \mathrm{dl}$ & $137 \mathrm{mg} / \mathrm{dl}$ & $137 \mathrm{mg} / \mathrm{dl}$ & $92 \mathrm{mg} / \mathrm{dl}$ \\
\hline Mediana da glicemia pós-prandial (mg/dl) & $145 \mathrm{mg} / \mathrm{dl}$ & $143 \mathrm{mg} / \mathrm{dl}$ & $205 \mathrm{mg} / \mathrm{dl}$ & $122 \mathrm{~g} / \mathrm{dl}$ \\
\hline \multirow[t]{2}{*}{ Malformações maiores } & -gônadas em fita & - membros curtos & - fenda palatina & $-\mathrm{CIV}$ \\
\hline & -hipoplasia pulmonar & & & $-\mathrm{CIA}$ \\
\hline \multirow[t]{5}{*}{ Malformações menores } & - agenesia renal & - implantação anormal do 3o & -nariz em sela & -imperfuração anal \\
\hline & & pododáctilo & -implantação baixa de & -hipospadia \\
\hline & & & orelhas & -micrognatia \\
\hline & & & -pescoço alado & -orelhas displásicas \\
\hline & & & -prega palmar única & -polidactilia \\
\hline
\end{tabular}

\section{Discussão}

Dentre as malformações em fetos de gestantes diabéticas, as maiores são as que tem um risco aumentado bem definido. Por definição, malformações maiores são aquelas que resultam em óbito ou morbidade prolongada ou que necessitem de correção cirúrgica. As que não se enquadram nessa categoria são consideradas malformações menores ${ }^{8}$.

Vários relatos mostram uma incidência elevada em 2 a 4 vezes de malformações maiores em conceptos de diabéticas ${ }^{11,12}$, em comparação com a população obstétrica normal, que está em torno de $1,7 \%$. Em relação a malformações menores, não parece haver uma maior incidência em gestantes diabéticas estando em torno de $1 \%{ }^{13}$. Em estudos anteriores, a avaliação das complicações maternofetais das diabéticas seguidas na nossa clínica revelou uma freqüência de malformações maiores que variou de 2,3 a $4 \%{ }^{14,15}$.

Apesar de a maioria dos trabalhos ter se baseado em gestantes diabéticas tipo 1 , justificada provavelmente pela menor ocorrência de gestações em diabéticas tipo 2, que incide em faixa etária 
mais avançada, tem sido demonstrada uma freqüência semelhante de MF nesse último grupo ${ }^{8}$.

No nosso estudo, encontramos, dentre os conceptos de mães diabéticas, 4 malformados $(11,4 \%)$. Todos tinham malformações maiores e somente um não apresentava associação com MF menores. É uma freqüência bem maior que a observada na população obstétrica normal e semelhante a descrita por outros autores em diabéticas.

O uso de sulfoniluréias em gestantes é ainda objeto de discussão. Enquanto em alguns países a sua utilização se faz rotineiramente, em sua maioria esses antidiabéticos orais têm sido proscritos no periodo gestacional. Sabendo-se que essas drogas atravessam a placenta, tem sido interrogado um possivel efeito teratogênico. Além disso, essas drogas têm um efeito estimulador sobre as células b fetais, elevando conseqüentemente os niveis de insulinemia fetal, podendo causar hipoglicemia neonatal de graus variáveis ${ }^{16}$.

Há várias décadas, a utilização de sulfoniluréias no período gestacional tem sido associada a malformações fetais. Além de vários relatos de casos de malformações em mulheres diabéticas em uso de sulfoniluréias durante a gestação, mais recentemente, Piacquadio et al. ${ }^{6}$ demonstraram $50 \%$ de $\mathrm{MF}$ em conceptos de diabéticas em uso de $\mathrm{SF}$, comparado a $15 \%$ encontrado no grupo controle, sugerindo também uma correlação entre o uso dessas drogas e malformações. Entretanto, essa correlação não ficou bem estabelecida em razão do mau controle glicêmico materno, fator que isoladamente já pode justificar a ocorrência de malformações. Em estudos animais, tem sido também sugerida essa associação ${ }^{17}$.

Observamos que os grupos, quando analisados quanto ao uso ou não de sulfoniluréias, mostraram uma freqüência estatisticamente semelhante de malformações, respectivamente 13,6 e $8,3 \%$ de MF maiores e 9,1 e 8,3\% de MF menores. Estes dados são concordantes com relato recente de Hellmuth et al. ${ }^{3}$, que, em estudo retrospectivo de 25 diabéticas tipo 2 que fizeram uso inadvertido de antidiabéticos orais durante a gestação, não encontraram maior prevalência de malformados, inclusive sem nenhum caso de malformação maior. Towner et al. ${ }^{8}$, em estudo prospectivo, no qual foram avaliadas, por meio de análise de regressão logística, 332 crianças de mães diabéticas tipo 2, também não encontraram correlação entre o uso dessas drogas e malformações. Demonstraram, no entanto, que essa complicação está relacionada com o mau controle glicêmico materno no período da concepção.

Fatores como dose, tempo de exposição e tipo de droga têm sido relacionados com os efeitos teratogênicos, não ficando, no entanto, estabelecida nenhuma correlação. Recentemente, Elliott et al. ${ }^{18}$ sugeriram haver variabilidade do transporte transplacentário das sulfoniluréias, sendo este menor para drogas de segunda geração.
Os RNs de duas pacientes que fizeram uso de clorpropamida e uma que usou glibenclamida apresentaram, respectivamente, as seguintes MF maiores: agenesia renal, gônadas em fita e hipoplasia pulmonar, membros curtos e fenda palatina. Essas MF não estão usualmente relacionadas com a embriopatia diabética, exceto a agenesia renal. No outro caso, em que o tratamento da mãe era feito com insulina, as MF maiores encontradas foram hipospadia, CIV e CIA e imperfuração anal, estando as 2 últimas entre as malformações usualmente observadas em filhos de diabéticas. As malformações cardiovasculares, do sistema nervoso central (SNC), do esqueleto axial, do trato urinário e as gastrointestinais têm sido especificamente encontradas em maior freqüência em RN de diabéticas $^{19,20}$. As MF cardiovasculares e do SNC parecem ser as mais freqüentemente associadas ao diabetes materno, sendo a sindrome de regressão caudal a mais específica.

Têm sido sugeridos alguns fatores maternos para explicar o maior risco de $\mathrm{MF}$ em fetos de diabéticas: fatores genéticos (ligados ao(s) gene(s) para diabetes), complicações crônicas do diabetes, como a microangiopatia, e anormalidades metabólicas. O controle metabólico materno inadequado tem sido apontado como o mais importante ${ }^{11,12}$. O mau controle metabólico das diabéticas, que ambos os grupos em nosso estudo apresentaram, já justificaria, portanto, as MF encontradas.

Como as MF encontradas nas pacientes que utilizaram as SF não estão entre as descritas caracteristicamente na embriopatia diabética, não é possivel descartar que possam ter sido devido à utilização dessas drogas.

Além disso, considerando-se não estar demonstrado que as sulfoniluréias são adequadas para obtenção de um controle metabólico adequado durante a gestação e o aumento no risco de hipoglicemia fetal com a sua utilização pela mãe, continuamos não prescrevendo esses antidiabéticos durante a gravidez.

O número de casos ora relatado e em estudos anteriores revela uma alta freqüência de gestações não-programadas. Com isso sugerimos uma maior atenção quanto ao aconselhamento pré-natal adequado das diabéticas em idade fértil.

\section{SUMMARY}

Purpose: to evaluate a possible relationship between fetal malformations (FM) and the use of sulfonylureas (SF) by diabetic pregnant women.

Methods: we retrospectively studied 35 type 2 diabetic pregnant women followed at the Pathological Prenatal Care Outpatient Clinic of the University Hospital, Faculty of Medicine of Ribeirão Preto, from 1993 to 1995. Twenty-two of these women had been inadvertently using sulfonylureas 
during the 1st trimester of gestation (SF group). We determined their prevalence of FM and compared it to that observed for pregnant diabetic women who were only on diet or insulin therapy (group C). We also analyzed other variables such as time of disease, age, metabolic control, and prenatal care. Results: there was no significant difference between groups in terms of age range, duration of diabetes, glycemic control, or early start of prenatal care, with the prevalence of FM being similar for the two groups (8.3\% in group C and $13.6 \%$ in group $S F)$. The malformations observed in group $S F$ were: renal agenesis, pulmonary hypoplasia and ribbon gonads (patient 1); short limbs and abnormally implanted toes (patient 2); cleft palate, low implanted ears, neck webbing and saddle nose (patient 3), and micrognathia, dysplastic ears, imperforate anus, hypospadia, polydactily, ventricular septal defect and atrial septal defect (patient 4) in group $C$.

Conclusions: these data do not allow us to attribute the malformations detected in group $S F$ to the use of sulfonylureas, although not usually described alterations in diabetic embryopathy occurred in this group.

KEY WORDS: Diabetes mellitus. Fetal malformations.

\section{Agradecimentos}

Ao Dr. Rafael Kioshi Yano, a Sra. Valéria Laguna Salomão Ambrósio (Serviço de Nutrição e Dietética), a Sra. Nadia Bittar Garcia e Sr. Sebastião Lázaro Brandão Filho.

\section{Referências}

1. Campbell GD. Possible teratogenic effect of tolbutamide in pregnancy. Lancet 1961; i: 891-2.

2. Dolger H, Bookman JJ, Nechemias C. The diagnostic and therapeutic value of tolbutamide in pregnant diabetics. Diabetes 1962; 11 (suppl): 97-8.

3. Hellmuth E, Damm P, Molsted-Pedersen L. Congenital malformations in offspring of diabetic women treated with oral hypoglycaemic agents during embryogenesis. Diabet Med 1994; 11: 471-4.

4. Jackson WPU, Campbell GD, Notelovitz M, Blumsohn D. Tolbutamide and chlorpropamide during pregnancy in human diabetics. Diabetes 1962; 11 (suppl): 98-103.

5. Larsson Y, Starky G. Possible teratogenic effect of tolbutamide in a pregnant diabetic. Lancet 1960; ii: $1424-5$.

6. Piacquadio K, Hollingsworth DR, Murphy H. Effects of in-utero exposure to oral hypoglycaemic drugs. Lancet 1991; 338: 866-9.

7. Schiff D, Aranda JV, Stern L. Neonatal thrombocytopenia and congenital malformations associated with administration of tolbutamide to the mother. J Pediatr 1970; 77: 457-8.
8. Towner D, Kjos SL, Leung B, Montoro MM, Xiang A, Mestman $\mathrm{JH}$, et al. Congenital malformations in pregnancies complicated by NIDDM. Diabetes Care 1995; 18: 1446-51.

9. De Veciana M, Major CA, Morgan MA, Asrat T, Toohey JS, Lien JM, et al. Postprandial versus preprandial blood glucose monitoring in women with gestational diabetes mellitus requiring insulin therapy. N Engl J Med 1995; 333: 1237-41.

10. Metzger BE. Summary and recommendations of the Third International Workshop-Conference on Gestational Diabetes Mellitus. Diabetes 1991; 40 (suppl 2): 197-201.

11.Kitzmiller JL, Gavin LA, Gin GD, Jovanovic-Peterson L, Main EK, Zigrang WD. Preconception care of diabetes. Glycemic control prevents congenital malformations. JAMA 1991; 265: 731-6.

12.Simpson JL, Elias S, Martin AO, Palmer MS, Ogata ES, Radvany RA. Diabetes in pregnancy, Northwestern University series (1977-1981). Prospective study of anomalies in offspring of mothers with diabetes mellitus. Am J Obstet Gynecol 1983; 146: 263-70.

13.Hod M, Merlob P, Friedman S, Litwin A, Mor N, Rusecki Y, et al. Prevalence of minor congenital anomalies in newborns of diabetic mothers. Eur J Obstet Gynecol Reprod Biol 1992; 44: 111-6.

14.Paccola GMGF, Torquato MTG, Baima Filho J, Duarte G, Foss MC. Diabetes Mellitus e Gravidez: Acompanhamento de 89 gestações de 1986 a 1991 no Hospital das Clínicas da Faculdade de Medicina de Ribeirão Preto - USP. Arq Bras Endocrinol Metab 1995; 39: 26-31.

15.Pimenta WP, Cunha SP, Foss MC. Gravidez e Diabetes Mellitus - Experiência do período de 1980 a 1985 no Hospital das Clínicas da Faculdade de Medicina de Ribeirão Preto, Universidade de São Paulo. Rev Bras Ginecol Obstet 1988; 3: 54-61.

16. Christesen HBT, Melander A. Prolonged elimination of tolbutamide in a premature newborn with hyperinsulinaemic hypoglycaemia. Eur J Endocrinol 1998; 138: 698-701.

17.Smoak IW. Teratogenic effects of tolbutamide on early-somite mouse embryos in vitro. Diabetes Res Clin Pract 1992; 17:161-7.

18.Elliott BD, Schenker S, Langer O, Johnson BS, Prihoda T. Comparative placental transport of oral hypoglycemic agents in humans: a model of human placental drug transfer. Am J Obstet Gynecol 1994; 171: 653-60.

19. Martinez-Frias ML. Epidemiological analysis of outcomes of pregnancy in diabetic mothers: identification of the most characteristic and most frequent congenital anomalies. Am J Med Genet 1994; 51: 108-13.

20.Ramos-Arroyo MA, Rodriguez-Pinnila E, Cordero JF. Maternal diabetes: the risk for specific birth defects. Eur J Epidemiol 1992; 8: 503-8. 